\title{
Management of ductal pancreatic cancer
}

\section{The oncologists view: systemic treatment options in 2018}

\author{
Angela Djanani · Andreas Schmiderer · Lukas Niederreiter (D) Markus Niederreiter · Herbert Tilg
}

Received: 15 January 2019 / Accepted: 13 March 2019 / Published online: 15 May 2019

(C) The Author(s) 2019

\begin{abstract}
Summary
Background The majority of patients diagnosed with pancreatic cancer have inoperable disease at the time of presentation. For this reason, systemic treatment is the cornerstone of therapy and more options for systemic therapy have now become available than were a decade ago.

Methods In this short review, we will give an overview on the systemic therapeutic landscape in 2018 and briefly discuss the challenging topic of neoadjuvant therapy.

Results However, there are a lot of unsolved questions and an urgent need of more clinical trials and new therapies.

Conclusions In conclusion, despite recent advances, there remains much room for improvement in all aspects of treatment for pancreatic cancer.
\end{abstract}

Keywords Ductal pancreatic cancer - Pancreas · Chemotherapy $\cdot$ FOLFIRINOX · Nanoliposomal irinotecan

\section{Introduction}

Ductal pancreatic cancer currently represents the seventh most common cause of cancer mortality worldwide, accounting for about $4 \%$ of cancer-related deaths in males and females [1]. It is more common in the elderly population and rarely diagnosed in people younger than 40 years of age. Unfortunately, less than $20 \%$ of patients present with localized, resectable, and

A. Djanani $(\bowtie) \cdot$ A. Schmiderer $\cdot$ L. Niederreiter .

M. Niederreiter $\cdot$ H. Tilg

Department of Internal Medicine I, Gastroenterology,

Hepatology, Metabolism \& Endocrinology, Medical

University Innsbruck, Anichstr. 35, 6020 Innsbruck, Austria

Angela.Djanani@i-med.ac.at hence potentially curable tumors. The overall 5-year survival rate among patients with pancreatic cancer is less than $5 \%[2,3]$. Based on current projections, pancreatic cancer is expected to be the second leading cause of cancer mortality in the US in the next 15 years [4]. Despite recent progress there remains much room for improvement in all aspects regarding the treatment of pancreatic cancer.

\section{Adjuvant therapy}

At the moment, standard treatment for patients with resectable pancreatic cancer is surgery followed by adjuvant chemotherapy. Because of the poor outcome associated with surgery alone, the role of adjuvant therapy has been extensively evaluated. The aim of systemic therapy is to reduce the risk of distant metastases.

A series of studies has established that 6 months of adjuvant chemotherapy with either infusional gemcitabine or infusional fluorouracil (5-FU) improves overall survival. The ESPAC-3 trial compared gemcitabine versus bolus 5-FU plus folinic acid. Median survival was equivalent, but gemcitabine was less toxic and therefore became standard of care for a long time [5]. In the recently published ESPAC-4 trial, adding capecitabine (oral Fluorouracil) to gemcitabine compared to gemcitabine improved median survival significantly (28.0 versus 25.5 months) [6]. Particularly R0-resected patients showed a marked benefit from combined chemotherapy (mOS 39.5 months). Given the results of ESPAC4 , this is now considered as the new standard of care, especially for the R0-resected group. However, $60 \%$ of the patients in this study were R1-resected and show a more confined benefit to a combined therapy with capecitabine and gemcitabine. 
Table 1 Selected ongoing neoadjuvant trials for PDAC

\begin{tabular}{|l|l|l|l|l|}
\hline Trial & Phase & Treatment & \multicolumn{2}{l}{ Endpoint } \\
\hline NEOPAC (NCT01521702) & 3 & Neoadj gem/ox + adj gem in resectable PCa & PFS \\
\hline NEOPA (NCT01900327) & 3 & Sequential neoadj CRT followed by surgery vs. surgery in resectable PC & 3-year survival \\
\hline NCT01458717 & 3 & Neoadj CRT vs. surgery in borderline resectable PC & 2-year survival \\
\hline NorPACT-1 (NCT02919787) & $2 / 3$ & Neoadj vs. adjuvant CT in resectable PA & OS after 1 year \\
\hline NCT02172976 & $2 / 3$ & Adjuvant gem vs. neoadj/adj FOLFIRINOX in resectable PCa & OS \\
\hline S1505 (NCT02562716) & 2 & Periop mFOLFIRINOX vs. gem/nab-paclitaxel in resectable PC & OS \\
\hline NCT03199144 & 2 & $\begin{array}{l}\text { Neoadj gem/nab-paclitaxel combined with radiotherapy in borderline } \\
\text { resectable PC }\end{array}$ & OS after 3a \\
\hline NCT02305186 & $1 / 2$ & CR +/- pembrolizumab in resectable and borderline PC & Safety/tumor infiltration \\
\hline PANDAS-PRODIGE 44 (NCT02676349) & 2 & Neoadj FOLFIRINOX +/-RCT in borderline PC & RO resection \\
\hline a years, CR/CRTChemoradiotherapy, OS overall survival; PA/PC pancreatic cancer, PFS progression free survival, RCT radiochemotherapy
\end{tabular}

Table 2 Selected ongoing phase III trials in mPDAC

\begin{tabular}{|c|c|c|c|c|}
\hline Study & $\begin{array}{l}\text { Mechanism of action of } \\
\text { experimental agent }\end{array}$ & Indication & Specific requirements & Trial number \\
\hline $\begin{array}{l}\text { Gemcitabine/nab-paclitaxel } \\
+/- \text { PEGPH2O }\end{array}$ & $\begin{array}{l}\text { PEGPH20: recombinant } \\
\text { hyaluronidase (stroma target) }\end{array}$ & 1st line metastatic & $\begin{array}{l}\text { High intratumoral levels of HA } \\
\text { (hyaluronic acid) }\end{array}$ & NCT02715804 \\
\hline $\begin{array}{l}\text { Gemcitabine/nab-paclitaxel } \\
\text { +/-BBI608 }\end{array}$ & $\begin{array}{l}\text { BBI608: cancer stem cell } \\
\text { inhibitor }\end{array}$ & 1st line metastatic & - & NCT02993731 \\
\hline F0LF0X +/-AM0010 & $\begin{array}{l}\text { AM0010: PEGylated human } \\
\text { IL-10 }\end{array}$ & $\begin{array}{l}\text { 2nd line (after gemc- } \\
\text { itabine-based treatment) }\end{array}$ & - & NCT02923921 \\
\hline Glufosfamide vs. 5-FU & $\begin{array}{l}\text { Glufosfamide: alkylating } \\
\text { agent }\end{array}$ & $\begin{array}{l}\text { 2nd line (after gemc- } \\
\text { itabine-based treatment) }\end{array}$ & - & NCT01954992 \\
\hline Olaparib vs. placebo & Olaparib: PARP inhibitor & $\begin{array}{l}\text { "Maintenance" treatment } \\
\text { following response or } \\
\text { stabilization on front-line } \\
\text { platinum }\end{array}$ & Germline $B R C A$ mutation & $\begin{array}{l}\text { NCT02184195 (results } \\
\text { expected in February 2019) }\end{array}$ \\
\hline
\end{tabular}

At the recent ASCO Annual Meeting, Conroy T. and colleagues presented the results of the phase III Unicancer GI PRODIGE 24/CCTG PA.6 trial, and in December 2018, the results were published in the NEJM [7]. Adjuvant treatment with modified FOLFIRINOX resulted in the longest overall survival yet reported for patients with resected pancreatic cancer [8]. With adjuvant modified FOLFIRINOX $(2400 \mathrm{mg}$ per square meter fluorouracil [5-FU], $400 \mathrm{mg}$ per square meter leucovorin, $150 \mathrm{mg}$ per square meter irinotecan, $85 \mathrm{mg}$ per square meter oxaliplatin) every 2 weeks, median overall survival was 54.4 months, compared with 35.0 months with gemcitabine. Similarly, patients with R1-resection margins had a significant benefit when treated with modified FOLFIRINOX. However, modified FOLFIRINOX is more toxic than gemcitabine. Adverse events of grade 3 or 4 occurred in $75.9 \%$ of the patients in the modified-FOLFIRINOX group and in $52.9 \%$ of those in the gemcitabine group. The toxic effects were reversible, except for oxaliplatin-induced peripheral neurotoxic effect.

Nevertheless, it should now be considered as the new standard for adjuvant therapy for patients who are able to tolerate it (young age und ECOG 0).

We expect this year the results of the APACT trial (gemcitabine/pemetrexed vs. gemcitabine). It will be very interesting to see the results of this study impact on our daily practice.

\section{Neoadjuvant approach}

The high rate of positive lymph nodes and surgical resection margins at the time of surgery has prompted investigators to evaluate preoperative chemoradiotherapy approaches. Moreover, real-life data demonstrate that only about $50 \%$ of the patients actually receive chemotherapy after resection [9]. In a recently published paper by Weinrich and colleagues, the rate of adjuvant chemotherapy was much higher (98\%) but only $50 \%$ of the patients completed the therapy (six cycles) [10]. The most common reason for discontinuation was toxicity.

Despite the use of neoadjuvant concepts in this patient population, scientific evidence remains scarce and no common practice has been established yet [11]. On the basis of their effectiveness in patients with metastatic disease (see below), there is growing interest in incorporating multiagent chemotherapy regimens such as the combination of fluorouracil, irinotecan, oxaliplatin, and leucovorin (FOLFIRINOX) and gemcitabine plus albumin-bound paclitaxel particles (nab-paclitaxel) in preoperative regimens. Interestingly, neoadjuvant FOLFIRINOX resulted in about 


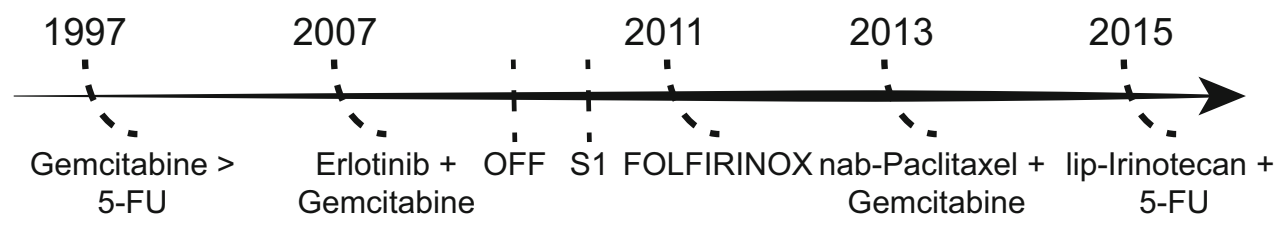

Fig. 1 Key milestones that represent significant advancements in the therapy of PDAC are shown in chronological order. 5-FU 5-Fluorouracil, FOLFIRINOX Oxaliplatin + Irinotecan + 5-Flourouracil, OFF Oxaliplatin + 5-Fluorouracil

$25 \%$ secondary resectability and a median survival of 24.2 months in a recent meta-analysis, making this chemotherapy a valuable tool for locally advanced disease [12].

At this year's ASCO-GI (2019), Unno M. et al. presented a Japanese phase II/III trial in resectable pancreatic cancer patients. One group (180 patients) was resected upfront and received adjuvant chemotherapy with $\mathrm{S} 1$, the other group $(n=182)$ received neoadjuvant chemotherapy with gemcitabine and S1, followed by surgery and adjuvant chemotherapy. Looking at their primary endpoint (mOS), the group with neoadjuvant therapy showed a mOS of 36.7 months vs. 26.6 months. This hints towards a potentially important role of neoadjuvant therapy in this patient group, but unfortunately the data cannot be easily transferred to other regions, since S1 is exclusively used in the Asian population and comparable studies using different treatment regimens are currently lacking.

At the moment there are ongoing trials to combine chemotherapy with radiotherapy in a neoadjuvant setting; however, firm conclusions cannot be drawn at this stage (Table 1).

Moreover, the use of neoadjuvant/preoperative therapy may result in progression of the disease in a proportion of patients to the point of becoming unresectable (missed window) and might increase the risk of perioperative morbidity and mortality due to side effects of chemotherapy or chemoradiation.

\section{Palliative therapy}

As mentioned above, more than half of the patients suffer from metastatic disease at the time of presentation. For these patients, palliative systemic treatment remains the only option in addition to best supportive care.

Fluorouracil-based chemotherapy compared to best supportive care alone improves survival by approximately 3 months [13]. In 1996, a study comparing gemcitabine with fluorouracil in patients with advanced pancreatic cancer showed an improvement in overall survival of roughly 1 month among patients receiving gemcitabine [14].

Over the next 10 years, multiple randomized studies compared single-agent gemcitabine with combination therapy. However, no combination regimen showed an improvement in overall survival.
Recently, two results from clinical phase III trials changed the standard of care from single-agent gemcitabine to combination chemotherapy in the firstline treatment strategy. The intense chemotherapeutic regime with FOLFIRINOX has been shown to be superior to gemcitabine monotherapy in PFS and OS [15]. In the MPACT trial, a combination of gemcitabine and nab-paclitaxel was also superior to singleagent gemcitabine [16].

Unfortunately, there has been no head-to-head comparison between these two regimes in a prospective randomized study. At the moment, there are no predictive biomarkers or patient subpopulations that allow to distinguish between patients who will benefit from one or the other regimen. However, pancreatic cancer associated with a BRCA2 mutation may be uniquely sensitive to platinum agents [17]. Patients who are known mutant BRCA carriers or have a strong family history of cancer suggesting $B R C A$ mutation should be considered for FOLFIRINOX. For all other patients, physicians have to decide which first-line therapy should be used on the basis of symptoms and general conditions.

About $40-48 \%$ of patients receive a second-line treatment. Most of the trials have focused on fluoropyrimidine-based combinations [18]. Positive results of the NAPOLI-1 trial, an FDA-approved, global randomized phase III study in patients, who had progressed on first-line gemcitabine-based chemotherapy, showed that the combination of nanoliposomal irinotecan with fluorouracil and folinic acid had a higher median overall survival compared to those receiving 5 -FU/leucovorin alone (6.1 vs. 4.2 months, HR 0.67, $p=0.012$ ) [19].

There are still ongoing trials for particular indications as well as for first-line therapy, and hopefully in the future there may be more options for patients requiring first- or second-line treatment (Table 2).

A small proportion of patients may even be suitable for third-line treatment, but scientific evidence and clinical trials are missing.

In conclusion, despite recent advances (Fig. 1), there remains much room for improvement in all aspects of treatment for pancreatic cancer.

Funding H. Tilg was supported by the excellence initiative (Competence Centers for Excellent Technologies: COMET) of the Austrian Research Promotion Agency FFG: Research Center of Excellence in Vascular Ageing Tyrol, VASCage (KProject no. 843536) funded by the BMVIT, BMWFW, the Wirtschaftsagentur Wien, and the Standortagentur Tirol. 
Funding Open access funding provided by University of Innsbruck and Medical University of Innsbruck.

Conflict of interest A. Djanani, A. Schmiderer, L. Niederreiter, M. Niederreiter, and H. Tilg declare that they have no competing interests.

Open Access This article is distributed under the terms of the Creative Commons Attribution 4.0 International License (http://creativecommons.org/licenses/by/4.0/), which permits unrestricted use, distribution, and reproduction in any medium, provided you give appropriate credit to the original author(s) and the source, provide a link to the Creative Commons license, and indicate if changes were made.

\section{References}

1. Are, Chowdhury, Ahmad, et al. Predictive global trends in the incidence and mortality of pancreatic cancer based on geographic location, socio-economic status, and demographic shift. JSurg Oncol. 2016;114(6):736-42.

2. Jemal, Siegel, Ward, et al. Cancer statistics. CA Cancer JClin. 2008;58(2):71-96.

3. Li, Xie, Wolff, et al. Pancreatic cancer. Lancet. 2004;363(9414):1049-57.

4. Rahib, Smith, Aizenberg, et al. Projecting cancer incidence and deaths to 2030: the unexpected burden of thyroid, liver, and pancreas cancers in the United States. Cancer Res. 2014;74(11):2913-21.

5. Neoptolemos, Stocken, Bassi, etal. Adjuvant chemotherapy with fluorouracil plus folinic acid vs gemcitabine following pancreatic cancer resection: a randomized controlled trial. JAMA. 2010;304(10):1073-81.

6. Neoptolemos, Palmer, Ghaneh, et al. Comparison of adjuvant gemcitabine and capecitabine with gemcitabine monotherapy in patients with resected pancreatic cancer (ESPAC-4): a multicentre, open-label, randomised, phase 3 trial. Lancet. 2017;389(10073):1011-24.

7. Conroy, Hammel, Hebbar, et al. FOLFIRINOX or gemcitabine as adjuvant therapy for pancreatic cancer. N Engl J Med. 2018;379(25):2395-406.

8. Conroy, Hammel, Hebbar, et al. Unicancer GI PRODIGE 24/CCTG PA.6 trial: A multicenter international randomized phase III trial of adjuvant mFOLFIRINOX versus gemcitabine (gem) in patients with resected pancreatic ductal adenocarcinomas. J Clin Oncol. 2018;36(18_suppl):LBA4001-LBA.

9. Bakens, van der Geest, van Putten, et al. The use of adjuvant chemotherapy for pancreatic cancer varies widely between hospitals: a nationwide population-based analysis. Cancer Med. 2016;5(10):2825-31.

10. Weinrich, Bochow, Kutsch, et al. High compliance with guideline recommendations but low completion rates of adjuvant chemotherapy in resected pancreatic cancer: A cohort study. Ann Med Surg (Lond). 2018;32:32-7.

11. Lutfi, Talamonti, Kantor, et al. Perioperative chemotherapy is associated with a survival advantage in early stage adenocarcinoma of the pancreatic head. Surgery. 2016;160(3):714-24.

12. Suker, Beumer, Sadot, et al. FOLFIRINOX for locally advanced pancreatic cancer: a systematic review and patientlevel meta-analysis. Lancet Oncol. 2016;17(6):801-10.

13. Sultana, Smith, Cunningham, et al. Meta-analyses of chemotherapy for locally advanced and metastatic pancreatic cancer. JClin Oncol. 2007;25(18):2607-15.

14. Burris, Moore, Andersen, et al. Improvements in survival and clinical benefit with gemcitabine as first-line therapy for patients with advanced pancreas cancer: a randomized trial. JClin Oncol. 1997;15(6):2403-13.

15. Conroy, Desseigne, Ychou, et al. FOLFIRINOX versus gemcitabinefor metastatic pancreatic cancer. NEnglJ Med. 2011;364(19):1817-25.

16. VonHoff, Ervin, Arena, etal. Increased survivalin pancreatic cancer with nab-paclitaxel plus gemcitabine. NEngl J Med. 2013;369(18):1691-703.

17. Golan, Kanji, Epelbaum, et al. Overall survival and clinical characteristics of pancreatic cancer in BRCA mutation carriers. Br JCancer. 2014;111(6):1132-8.

18. Von Hoff, Ramanathan, Borad, et al. Gemcitabine plus nab-paclitaxel is an active regimen in patients with advanced pancreatic cancer: a phase I/II trial. J Clin Oncol. 2011;29(34):4548-54.

19. Wang-Gillam, Li, Bodoky, et al. Nanoliposomal irinotecan with fluorouracil and folinic acid in metastatic pancreatic cancerafter previousgemcitabine-based therapy (NAPOLI1): a global, randomised, open-label, phase 3 trial. Lancet. 2016;387(10018):545-57. Feb.

Publisher's Note Springer Nature remains neutral with regard to jurisdictional claims in published maps and institutional affiliations. 